\title{
Maturity Onset Diabetes of the Young (MODY)
}

\author{
S.S. Fajans ${ }^{a}$, G.I. Bell', D.W. Bowdenc, J.B. Haltera, K.S. Polonskyd \\ -Department of Internal Medicine, University of Michigan Medical \\ Center and the Department of Veterans Affairs Medical Center, Ann \\ Arbor, Michigan, 'The Howard Hughes Medical Institute, University \\ of Chicago, Chicago, llinois, "Department of Biochemistry, Bowman \\ Gray School of Medicine, Wake Forest University, Winston-Salem, \\ North Carolina, and Department of Medicine, University of \\ Chicago, llinois, USA
}

\begin{abstract}
MODY is a sub-type of NIDDM. It is characterized by an early age of onset and autosomal dominant mode of inheritance. These features, and the availability of large multigenerational pedigrees, make MODY useful for genetic studies of diabetes. In the large 5-generational RW pedigree, MODY is tightly linked to genetic markers on chromosome $20 \mathrm{q}$. Affected subjects in this family show abnormalities of carbohydrate metabolism, varying from impaired glucose tolerance (IGT) to severe diabetes. Approximately $30 \%$ of diabetic subjects become insulin-requiring, and vascular complications occur. MODY is also linked to the glucokinase gene on chromosome $7 p$ and many different mutations associated with MODY have been identified in this gene. MODY, due to mutations in the glucokinase gene, is a relatively mild form of diabetes with mild fasting hyperglycaemia and IGT in the majority. Clinical investigative studies indicate that the genetic or primary defect in MODY is characterized by deranged and deficient insulin secretion and not by insulin resistance. There are quantitative and qualitative differences in insulin secretory defects which differentiate subjects with MODY due to mutation in the gene on chromosome 20q from those with glucokinase mutations. These differences correlate with the severity of diabetes between these two genetic forms of MODY.
\end{abstract}

KEY WORDS Maturity-onset diabetes of the young (MODY) Genetics of diabetes Insulin secretion Insulin resistance

\section{Introduction}

Maturity onset diabetes of the young (MODY), a relatively uncommon subtype of NIDDM, is a valuable model for the study of the molecular genetics and pathogenesis of NIDDM. ${ }^{1,2}$ A previous review summarized our expanding knowledge of the phenotypic expression, natural history, molecular genetics and pathogenesis of MODY. ${ }^{2}$ This presentation gives a condensed version of the previous review; it includes additional data on insulin secretion.

\section{Definition, Phenotypic Expression, and Natural History}

MODY is defined as NIDDM characterized by an early age of onset and autosomal dominant mode of inheritance. ${ }^{1-4}$ When MODY is suspected and family members are examined biochemically, a diagnosis of diabetes can be made almost invariably under the age of 25 years, frequently between the ages of 9 and 13 years, and in some families at an even earlier age. Because of autosomal dominant inheritance and early age of onset it is possible to collect large multigenerational

Correspondence to: Dr Stefan S. Fejans, 3290 Taubman Center, Box 0354, University of Michigan Medical Center, Ann Arbor, MI 481090354, USA. pedigrees with MODY, a unique feature of this subtype of NIDDM.

The RW pedigree, consisting of more than 360 presently identified members including 72 known subjects with diabetes distributed over 5 generations, has been studied and followed prospectively by one of us (SSF) since 1958. The propositus, II-5 (W branch), offspring of I-3 had diabetes diagnosed at the age of 41 years (Figure $1(a))$. He was blind from diabetic retinopathy at the age of 61 years and had an amputation for peripheral vascular disease. He had five brothers, four of whom had diabetes and one who did not. Macrovascular disease including myocardial infarctions and peripheral vascular disease with gangrene and amputations, as well as microvascular disease including retinopathy and blindness are complications resulting from MODY in the RW pedigree (Figure 1). In addition, there is also evidence of neuropathy. In 1958, the 11 non-obese, apparently healthy and asymptomatic offspring of II-5 were recruited for routine blood glucose testing. Seven of the 11 were found to have abnormal glucose metabolism. ${ }^{3}$ The older 3 had fasting hyperglycaemia (mean $257 \mathrm{mg} \mathrm{dl}^{-1}$ ), 3 others had diabetic glucose tolerance tests without diagnostic fasting hyperglycaemia, and 1 had IGT.

With follow-up, all have developed fasting hyperglycaemia. Two have been treated with insulin because of eventual unresponsiveness to sulphonylurea drugs, 4 
PEDIGREE R-W

A

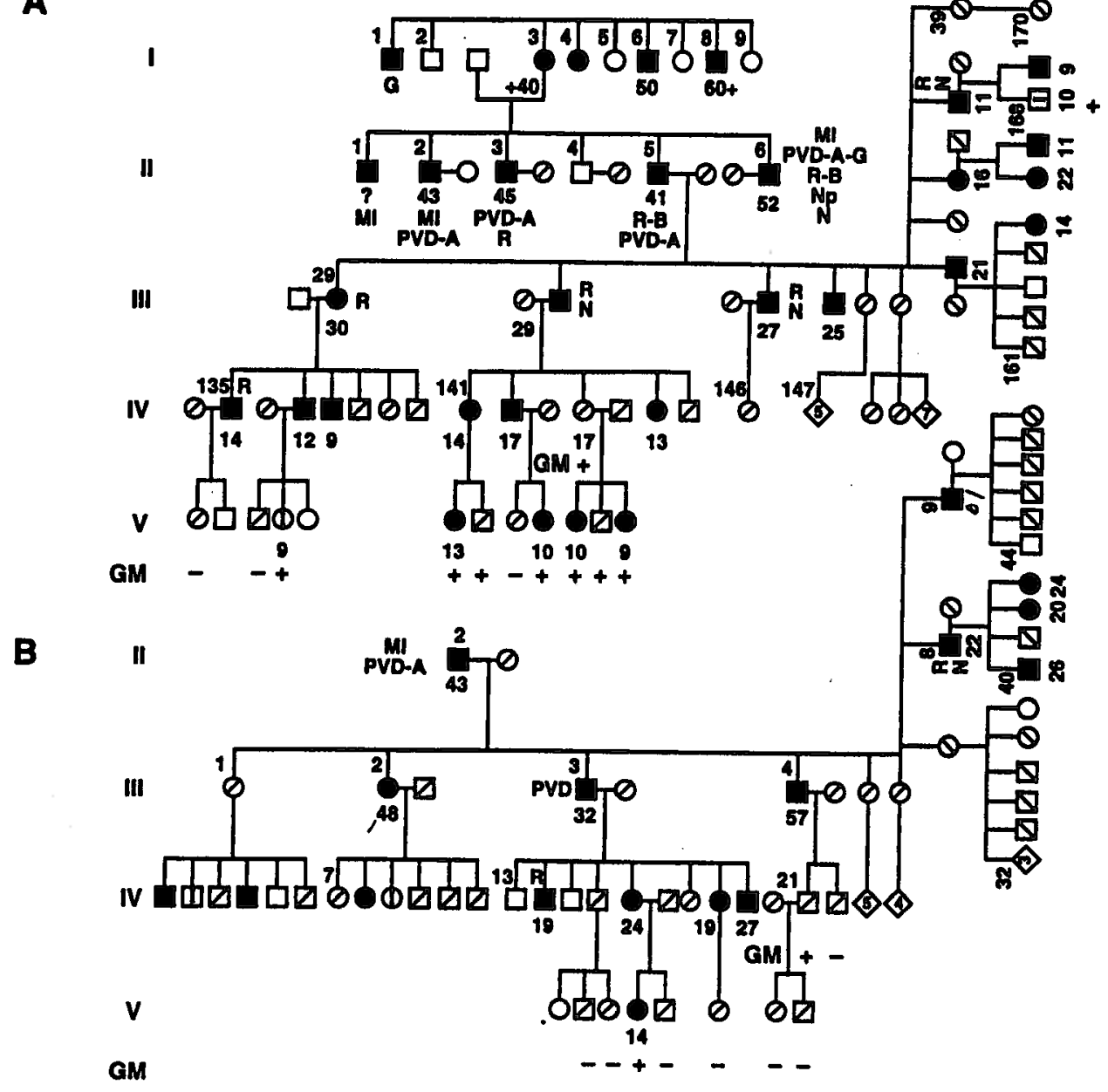

Figure 1. Partial pedigree of the RW family. Offspring of $1-1$ belong to the R branch. Offspring of 1-3 belong to the W branch. Only the offspring of II-5 (a) and II-2 (b) of the W branch are shown. All subjects with diabetes were or are non-insulin-dependent except IV-144 who is insulin-dependent. Diabetic or IGT offspring of II-5 of generations III-V (a) and diabetic offspring III-3 and III-8 (b) and their diabetic offspring are genetic marker (chromosome 20) positive. In addition, nondiabetic IV-143 (a) and IV-21 (b) are genetic marker $(\mathrm{GM})$ positive $(+)$. In generation $V, G M+$ or negative $(-)$ are indicated $(a, b)$. Numbers above male diabetic members $(\square)$ or female diabetic members $(\bullet)$ are number in generation and those below are ages at diagnosis. l, impaired glucose tolerance; $\varnothing$ normal glucose tolerance; $\square$ reported normal and untested; $\diamond$ groups of siblings untested and of unspecified sex with number of individuals given; MI, myocardial infarction; PVD, peripheral vascular disease; $A$, amputation; $G$, gangrene; $N$, neuropathy; Ang, angina pectoris; R, retinopathy; R-B, retinopathy and blindness; NP, nephropathy. All diabetic members of generations I and II, along with III-29 and III-30, are deceased. (From Fajan et al. ${ }^{2}$ with kind permission of Elsevier Science Ltd, Kidlington, Oxford, UK.)

have been treated successfully with sulphonylurea drugs, up to the present, and 1 is using diet alone, although sulphonylurea therapy is indicated because he has recently developed fasting hyperglycaemia. Subsequently, by routine blood glucose testing diabetes was diagnosed in 10 of the 20 members of generation IV who are offspring of diabetic subjects of generation III, and up to the present time in 4 members of generation V. None of them are obese. Ages of diagnosis were 914 years in 12 of these patients with diabetes. Patient IV-144 has typical IDDM. Among offspring of II-2, 3 of the 5 diabetic members (III-3, III-4, III-8) have MODY Segregating in generations III-V (Figure 1(b)). I-1 (Figure 1 (a)) had 3 offspring, 2 of whom had diagnosed diabetes ( $R$ branch, Generation II, not shown in Figure 1). Among their 10 offspring in generation III, 6 have or have had diagnosed NIDDM. Of 12 tested subjects in Generation IV who are offspring of a diabetic parent, 5 have diabetes. Thus, diabetes is inherited in the autosomal pattern of MODY in the $R$ branch as well. None of them are obese.

MODY is usually asymptomatic in younger age groups, although some patients may have symptoms, particularly if stressed by an infection. Unless searched out by prospective testing, because of a family history of NIDDM in two or more generations, in a young person or during a pregnancy, a clinical diagnosis of diabetes is frequently not made in many members of such families until middle or late adult life. Thus, age of diagnosis cannot be equated to age of onset of hyperglycaemia. Younger family members must be screened before typical MODY 
can be excluded. As demonstrated in the RW pedigree in which MODY is linked to chromosome 20q (see below), it can be demonstrated by repeated testing in younger members that there may be a variable rate of progression from nondiagnostic (but not normal) glucose tolerance tests to IGT, from IGT to diabetic glucose tolerance with normal fasting plasma glucose levels (up to 18 years), and very slow or rapid progression to fasting hyperglycaemia ( 0.5 to 27 years). In contrast, other individuals with MODY from this pedigree may have fasting hyperglycaemia or rapid progression from an early age, even though they are asymptomatic. Among nonobese diabetic subjects of the RW pedigree in generations III-V approximately $80 \%$ have had fasting hyperglycaemia at diagnosis or on follow-up. Plasma glucose levels at first detection of fasting hyperglycaemia range between 140 and $366 \mathrm{mg} \mathrm{dl}^{-1}$. Approximately $30 \%$ had fasting hyperglycaemia diagnosed between the ages of 9 and 14 years or within a 1 to 3 year followup. Among the known diabetic patients from generations III-V of the RW pedigree approximately $30 \%$ have become insulin-requiring. Typical microangiopathic and macroangiopathic complications may occur similar to those seen in other patients with NIDDM..$^{3,4}$

in contrast to MODY linked to chromosome $20 \mathrm{q}$, as present in the RW pedigree, MODY due to mutations in the glucokinase gene on chromosome $7 p$ (see below) is a relatively mild form of diabetes with mild fasting hyperglycaemia and IGT in the majority that may be recognized by biochemical testing at an even younger age (mean $7 \pm 4$ years, minimal age, 1 year). ${ }^{5}$ There appears to be little progression of hyperglycaemia on follow-up..$^{6,7}$ Severe fasting hyperglycaemia and vascular complications appear to be infrequent (Table 1). Thus MODY due to glucokinase mutations appears to be a much milder and relatively benign form of hyperglycaemia, in contrast to the more severe diabetes seen in MODY linked to chromosome $20 q$ that more closely resembles classical NIDDM.

\section{Molecular Genetics of MODY}

MODY fulfils some of the fundamental requirements for defining the molecular genetic basis of any disorder by linkage analysis because of its established autosomal dominant inheritance and the availability of large multigenerational kindreds. The RW pedigree was first used for a search of diabetes susceptibility genes by this technique. The initial linkage strategy was to search for 'candidate genes', that is genes involved in carbohydrate and lipid metabolism. Such genes were excluded as the cause of NIDDM in the RW pedigree. ${ }^{8}$ In a subsequent systematic gene mapping approach (positional cloning), DNA polymorphisms in the adenosine deaminase gene (ADA) and the anonymous locus D20S16 on the long arm of chromosome 20 were found to cosegregate with MODY ${ }^{8-13}$ in members with diabetes of both $W$ and $R$ branches. There are no recombinations between these markers and MODY and the lod score presently exceeds 17.0 at a recombination fraction of 0.00 , indicating that the gene responsible for MODY in this family is tightly linked to these genes.

The identification of genetic markers for MODY in the RW pedigree has shown that linkage studies of large MODY families can uncover DNA markers for NIDDM..$^{8,10}$ The gene responsible for MODY in the RW pedigree is unknown but is in the region of $A D A$ and D20S16 on chromosome 20q. DNA typing can identify 'at risk' subjects, i.e. genetic screening for prediabetes is possible in the RW pedigree. Identification of the

Table 1. Differences between MODY due to mutations in the glucokinase gene (chromosome $7 p$ ) and the diabetes-susceptibility gene on chromosome $20 q$

Glucokinase gene

$\begin{array}{ll}0-++ & 0-++++ \text { (approx. 80\%) } \\ 0-++ & ++-++++ \\ 0 & ++-++++ \\ 1 \text { year } & 7-9 \text { years } \\ \text { Uncommon (2\%) } & \text { Common (approx. 30\%) } \\ \text { Rare } & ++ \\ \text { Normal }-\downarrow & \downarrow \downarrow-\text { absent }\end{array}$

Fasting hyperglycaemia (>140 $\mathrm{mg} \mathrm{dl}^{-1}$ )

Postprandial hyperglycaemia

Progression of hyperglycaemia

Minimum age at diagnosis

Need for insulin therapy

Vascular complications

AlRglu*

ISR $^{\mathbf{b}}$ during graded IV glucose infusion: mildly diabetic MODY

nondiabetic subjects with genetic markers

Glucose priming of ISR

MODY

nondiabetic subjects with genetic markers

Postulated abnormality in $\beta$-cell function $\downarrow-\downarrow \downarrow$

Not available

Normal (45\% increase)

Not available

Glucose sensing
Chromosome $20 \mathrm{q}$

$\downarrow \downarrow$

$\downarrow \downarrow$

Absent

Absent

Locus beyond glucose sensing or decrease in $\beta$-cell mass 
MODY gene on chromosome 20 may disclose previously unrecognized mechanisms controlling insulin secretion and lead us closer to an understanding of the pathogenesis of MODY in this pedigree, with possible application to NIDDM in general. Such studies may lead eventually to the prevention, improved treatment, or even a cure for this and other forms of NIDDM.

Because there is heterogeneity between MODY families by hormonal and metabolic characterization, ${ }^{14}$ it was postulated that additional NIDDM susceptibility genes or modifying determinants would be found. ${ }^{8}$ Indeed, this occurred shortly thereafter. Tight linkage between MODY and the glucokinase gene on chromosome $7 p$ was found in approximately $60 \%$ of 32 French MODY families, ${ }^{5,15}$ in one British family ${ }^{6,16}$ and two in Japanese families. ${ }^{17,18}$ Glucokinase is the first rate-limiting enzyme in the metabolism of glucose by the pancreatic $\beta$-cell. It causes the phosphorylation of glucose to glucose-6-phosphate initiating glycolysis which is essential for insulin secretion. This enzyme has been postulated to be the glucose sensing mechanism for insulin secretion. ${ }^{19}$ Subsequently, a host of different mutations (nonsense, missense or deletions) in the glucokinase gene have been found in members of MODY families in which MODY is linked to the glucokinase gene. ${ }^{5,16-18,20-23}$ They are believed to cause diabetes by a gene dosage mechanism with a modest decrease in glucokinase activity increasing the glucose threshold for insulin secretion. Matschinsky has suggested that a $15 \%$ decrease in glucokinase activity may raise the threshold of insulin secretion from 5 to 6 mmol. ${ }^{19}$ Mutations in the glucokinase gene are the most common genetic cause of NIDDM identified to date, particularly among MODY pedigrees. The RW pedigree is the only large pedigree in which tight linkage of MODY to chromosome $20 \mathrm{q}$ has been established. Evidence consistent with linkage to chromosome 20q has been found in two French ${ }^{5,15}$ and one Canadian (D.W.Bowden, E, Colle, unpublished results) MODY pedigrees.

In addition to the glucokinase gene and the gene on chromosome 20, there must be other loci that can cause MODY as MODY pedigrees have been described that show no evidence of linkage with glucokinase or markers on chromosome $20.5,6,11,15,24$

\section{$\beta$-Cell Function and Insulin Resistance}

In the RW pedigree, the majority of MODY subjects who were offspring of $11-2$ and $11-5$ have a delayed and subnormal insulin secretory response to orally administered glucose, strongly suggesting an impairment of $\beta$-cell function. In these individuals impaired $\beta$-cell function, although moderate in magnitude, appears to be the major underlying pathogenetic factor for abnormal glucose levels. The low insulin secretory response to glucose may occur from childhood onwards and before glucose intolerance appears. This is demonstrated by insulin levels during the oral glucose tolerance test in nondiabetic subjects who are genetic marker negative or positive (Figure 2). The nondiabetic marker-negative subjects had a mean insulin response to the administration of glucose which was almost identical to that previously reported in 150 control subjects without a family history of diabetes. ${ }^{14}$ In contrast, nondiabetic subjects who are marker-positive had a decreased insulin response to glucose, maximal levels at $1 \mathrm{~h}$ being approximately onehalf of that of the marker-negative subjects. Subjects with IGT had a similar or slightly lower insulin response to glucose. Other groups of subjects with IGT have been reported to have normal or elevated levels of plasma insulin during the OGTT as compared to control subjects. ${ }^{25,26}$ MODY subjects with fasting plasma glucose levels below $140 \mathrm{mg} \mathrm{dl}^{-1}$ showed a further decline in the insulin response with additional progressive decreases in diabetic subjects with fasting plasma glucose concentrations between 140 and 200 and greater than 200 $\mathrm{mg} \mathrm{dl}^{-1}$ (Figure 2). The defective insulin secretory response was postulated to be a manifestation of the basic genetic defect that leads to diabetes only when additional superimposed environmental factors supervene., ${ }^{3,4}$ Pathological insulin resistance does not appear to be an important contributing factor because of normal sensitivity to intravenously administered insulin and because of the usually low insulin requirement in insulintreated diabetic members of the RW pedigree.

In diabetic MODY patients linked to a mutation in the glucokinase gene, there is evidence of decreased insulin secretion by continuous glucose infusion ${ }^{6,7,27}$ although the acute insulin response to glucose was within normal limits. ${ }^{7,27}$ A decreased insulin secretory response to orally administered glucose has also been reported in glucokinase-linked MODY. ${ }^{18}$ In addition, no evidence of insulin resistance has been reported ${ }^{7,27}$ in such patients. This is in contradistinction to patients with other types of NIDDM, most of whom are obese, in whom it has been found or postulated that insulin resistance is a more important factor than insulin deficiency in the pathogenesis of diabetes. ${ }^{28,29}$

Since chronic hyperglycaemia has been reported to be associated with both a decrease of insulin sensitivity as well as an impairment of $\beta$-cell function, the question of which is the primary genetic defect in MODY or NIDDM is very difficult to address in diabetic patients. ${ }^{28}$ Since we now have genetic markers for MODY in the RW pedigree, we undertook to determine early abnormalities of insulin action and of insulin secretion in nondiabetic subjects of the pedigree with and without the gene markers to ascertain which of these defects might be primary. Six nondiabetic marker-negative and 5 nondiabetic marker-positive members of the RW pedigree were studied, as were 4 mildly diabetic markerpositive family members. Unrelated, young, healthy subjects served as comparison groups. ${ }^{30}$ Insulin action and insulin secretion were assessed with a frequently sampled intravenous glucose tolerance test (Bergman Minimal Model). Insulin secretion was further assessed 

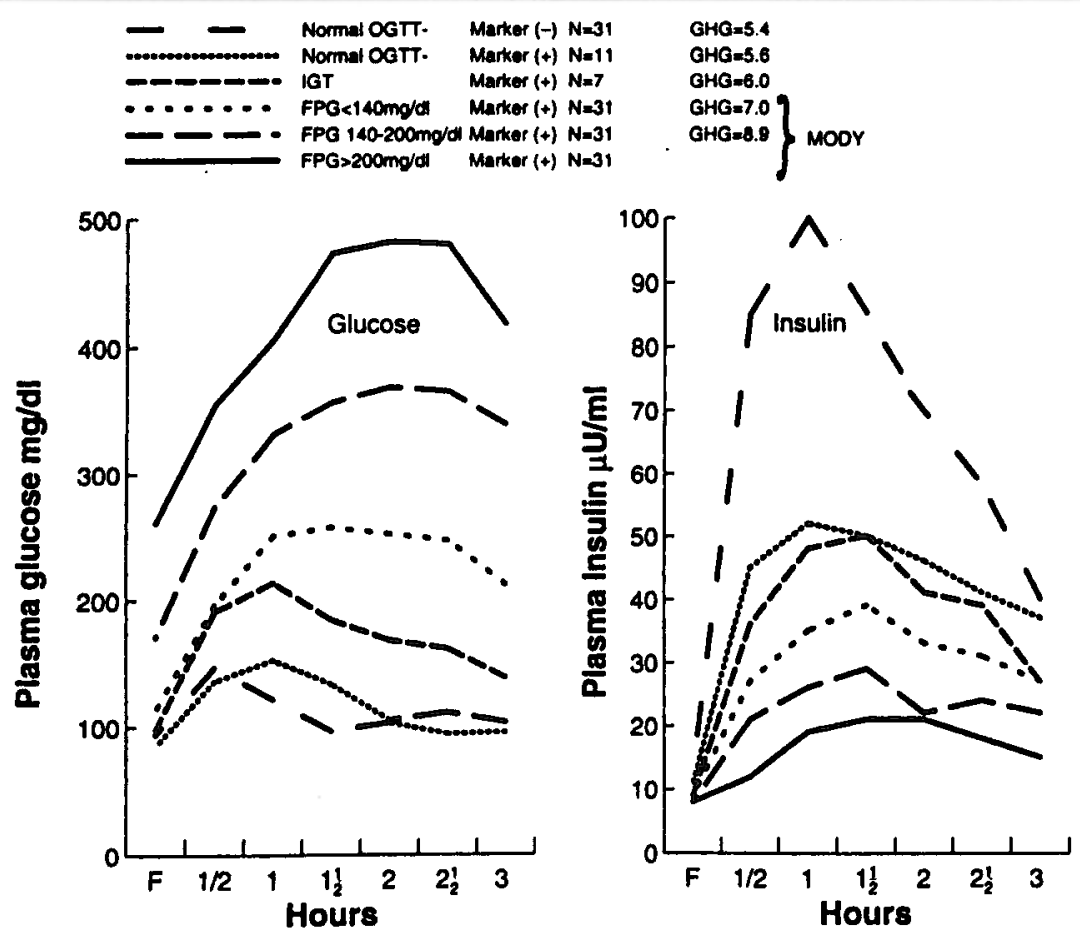

Figure 2. Plasma concentrations of glucose and insulin during oral glucose tolerance tests (1.75 $\mathrm{g} \mathrm{kg}^{-1}$ body weight) in groups of subjects of the RW pedigree

during constant low dose glucose infusion by deconvolution of plasma C-peptide and by pulse analysis.

The non-diabetic marker-positive group had normal sensitivity to insulin and unimpaired acute insulin response to intravenous glucose. However, the nondiabetic marker-positive group had decreased mean plasma C-peptide concentration, reduced absolute amplitude of insulin secretory ultradian oscillations, and decreased insulin secretion rate during prolonged glucose infusion. $^{2,30}$ These responses to prolonged glucose infusion were similar to those observed in the diabetic group, who, in addition, also had a decreased or absent acute insulin response to intravenous glucose. ${ }^{30}$ No alterations of insulin secretion were observed in the nondiabetic marker-negative family members or in the comparison groups. Deranged and deficient insulin secretion, and not insulin resistance, appears to be the genetic or primary abnormality that characterizes nondiabetic individuals who are predisposed to MODY in the RW pedigree. Prolonged glucose infusion studies may reveal qualitative and quantitative defects in insulin secretion not identified by the acute insulin response to intravenous glucose. Furthermore, use of the acute insulin response to glucose may not be able to exclude a primary $\beta$-cell defect in the pathogenesis of any form of NIDDM. ${ }^{30}$

Quantitative and qualitative differences in insulin secretory defects between MODY with glucokinase mutations and MODY with mutation on chromosome $20 q$ have been found by studies with acute and more prolonged intravenous infusions of glucose. MODY subjects with glucokinase mutations have a first phase insulin response to glucose which is in the normal range or only slightly decreased 7,27 while mildly diabetic patients with a chromosome 20 mutation have a decreased or absent acute response to glucose. ${ }^{30}$ During stepped glucose infusions, both groups of MODY subjects (including nondiabetic marker-positive subjects of the RW pedigree) showed a decreased response of the $\beta$ cell to glucose resulting in a downward shift in the glucose/insulin secretion rate dose-response curve to the right which was more severe in RW subjects with a failure of $\beta$-cell sensitivity to improve after prolonged priming with intravenous glucose. 27,31

Although the specific genetic defect causing MODY in the RW pedigree is unknown, the quantitative and qualitative differences in insulin secreting defects in these subjects suggests a different mechanism of the $\beta$-cell dysfunction which may distinguish this form of MODY from that due to mutations in the glucokinase gene (Table 1). The greater severity of the insulin secretory defect in mildly diabetic and nondiabetic subjects with mutation in the diabetes susceptibility gene on chromosome $20 \mathrm{q}$ correlates with a greater severity of hyperglycaemia, a greater need for insulin therapy and a greater prevalence of vascular complications seen in these diabetic subjects than in those with mutations in the glucokinase gene (Table 1).

\section{Acknowledgements}

This work was supported by grants from the National Institutes of Health to the Michigan Diabetes Research 
and Training Center (DK-20572), the Claude D.Pepper Geriatric Research Center (AG-08808), the University of Michigan General Clinical Research Center (MO1RR00042), the Medical Research Service of the Department of Veterans Affairs, RO1-DK41269 (D.W.B) and the University of Chicago Diabetes Research and Training Center (DK-20595), Howard Hughes Medical institute, General Clinical Research Center (RR-00055) and RO1DK31842 (K.S.P.), The University of Chicago.

\section{References}

1. Fajans SS, Bell GI, Bowden DW. MODY: a model for the study of the molecular gentics of NIDDM. J Lab Clin Med 1992; 119: 206-210.

2. Fajans SS, Bell GI, Bowden DW, et al. Minireview: maturity-onset diabetes of the young. Life Sciences 1994; 55: 413-422.

3. Fajans SS. Maturity-onset diabetes of the young (MODY). Diabetes/Metabolism Reviews 1989; 5: 579-606.

4. Fajans SS. Scope and heterogeneous nature of MODY. Diabetes Care 1990; 13: 49-64.

5. Froguel $P$, Zoulai $H$, Vionnet $N$, et al. Familial hyperglycaemia due to mutations in glucokinase: definition of a subtype of diabetes mellitus. N Engl / Med 1993; 328: 697-702.

6. Hattersley AT, Turner RC, Permutt MA, et al. Linkage of type 2 diabetes to the glucokinase gene. Lancet 1992; 339: $1307-1310$.

7. Velho G, Froguel P Clement K, et al. Primary pancreatic beta-cell secretory defect caused by mutations in glucokinase gene in kindred of maturity onset diabetes of the young. Lancet 1992; 340: 444-448.

8. Bell Gl, Xiang K, Newman MC, et al. Gene for non-insulindependent diabetes mellitus (maturity-onset diabetes of the young subtype) is linked to DNA polymorphism on human chromosome 20q. Proc Natl Acad Sci USA 1991; 88: 1484-1488.

9. Cox NJ, Xian K, Fajans S, et al. Mapping diabetessusceptibility genes: lessons learned from search for DNA marker for maturity-onset diabetes of the young. Diabetes 1992; 41: 401-407.

10. Bowden DW, Gravius TC, Akots G, et al. Identification of genetic markers flanking the locus for maturity-onset diabetes of the young on human chromosome 20. Diabetes 1992; 41: 88-92.

11. Bowden DW, Akots G, Rothschild CB, et al. Linkage analysis of maturity-onset diabetes of the young (MODY): genetic heterogeneity and non penetrance. Am J Hum Genet 1992; 50: 607-618.

12. Rothschild CB, Akots G, Fajans SS, et al. A microsatellite polymorphism associated with PLC1 (phospholipase C) locus: identification, mapping, and linkage to the MODY locus on chromosome 20. Genomics 1992; 13: 560-564.

13. Rothschild CB, Akots $G$, Hayworth $R$, et al. A genetic map of chromosome 20q12-q12.1: multiple highly polymorphic microsatellite and RFLP markers linked to the maturity-onset diabetes of the young (MODY) locus. Am J Hum Genet 1993; 52:110-123.

14. Fajans SS. MODY - a model for understanding the pathogeneses and natural history of type II diabetes. Horm Metabol Res 1987; 19: 591-599.
15. Froguel $P$, Vaxillaire $M$, Sun $F$, et al. Close linkage of glucokinase locus on chromosome $7 p$ to early-onset noninsulin-dependent diabetes mellitus. Nature 1992; 356: 162-164.

16. Stoffel $M$, Patel $P$, Loy $Y$, et al. Missense glucokinase mutation in maturity-onset diabetes of the young and mutation screening in late-onset diabetes. Nature Genetics 1992; 2: 153-156.

17. Sakura $H$, Eto $K$, Kadowaki $H$, et al. Structure of the human glucokinase gene and identification of a missense mutation in a lapanese patient with early-onset noninsulin-dependent diabetes mellitus. J Clin Endocrinol Metab 1992; 75: 1571-1573.

18. Shimada F, Makino $H$, Hashimoto $N$, et al. Type 2 (noninsulin-dependent) diabetes mellitus associated with a mutation of the glucokinase gene in a Japanese family. Diabetologia 1993; 36: 433-437.

19. Matschinsky F. Glucokinase as glucose sensor and metabolic signal generator in pancreatic $\beta$-cells and hepatocytes. Diabetes 1990; 39: 647-652.

20. Vionnet $N$, Stoffel $M$, Takeda J, et al. Nonsense mutation in the glucokinase gene cause early-onset non-insulindependent diabetes mellitus. Nature $1992 ; 356$ : 721-722.

21. Stoffel $M$, Froguel $\mathrm{Ph}$, Takeda J, et al. Human glucokinase gene: isolation, characterization, and identification of two missense mutations linked to early-onset non-insulindependent (type 2) diabetes mellitus. Proc Natl Acad Sci USA 1992; 89: 7698-7702.

22. Sun F, Knebelmann B, Pueyo ME, et al. Deletion of the donor splice site of Intron 4 in the glucokinase gene causes maturity-onset diabetes of the young. / Clin Invest 1993; 92: 1174-1180.

23. Gidh-Jain M, Takeda J, Xu LZ, et al. Glucokinase mutations associated with non-insulin-dependent (type 2) diabetes mellitus have decreased enzymatic activity: implications for structure/function relationships. Proc Natl Acad Sci USA 1993; 90: 1932-1936.

24. Vaxillaire $M$, Vionnet $N$, Vigouroux $C$, et al. Search for a third susceptibility gene for maturity-onset diabetes of the young. Diabetes 1994; 43: 389-395.

25. Reaven $G$, Vernstein $R$, Davis B, et al. Noketotic diabetes mellitus: insulin deficiency or insulin resistance? $A m$ J Med 1976; 60: 80-88.

26. Taylor SI, Accili D, Imai Y. Insulin resistance or insulin deficiency: which is the primary cause of NIDDM? Diabetes 1994; 43: 735-740.

27. Byrne MM, Sturis J, Clement $K$, et al. Insulin secretory abnormalities in subjects with hyperglycaemia due to glucokinase mutations. J Clin Invest 1994; 93: 1120-1130.

28. De Fronzo RA, Bonadonna RC, Ferrannini E. Pathogenesis of NIDDM: a balanced overview. Diabetes Care 1992; 15: 318-368.

29. Lillioja S, Mott DM, Spraul $M$, et al. Insulin resistance and insulin secretory dysfunction as precursors of noninsulin-dependent diabetes mellitus. N Eng/ / Med 1993; 1988-1992.

30. Herman WH, Fajans SS, Ortiz F\}, et al: Abnormal insulin secretion, not insulin resistance, is the genetic or primary defect of MODY in the RW pedigree. Diabetes 1994; 43: 40-46.

31. Byrne MA, Sturis J, Fajans SS, et al. Altered insulin secretory responses to glucose in subjects with a mutation in the MODY1 gene on chromosome 20. Diabetes 1995; 44: 699-704. 